\title{
Eye Tracker as a Tool for Engineering Education
}

\author{
Kavin Kathiresh Vijayan", Ola Jon Mork, Irina Emily Hansen \\ Department of Ocean Operations and Civil Engineering, Norwegian University of Science and Technology (NTNU), Norway
}

Copyright $\bigcirc 2018$ by authors, all rights reserved. Authors agree that this article remains permanently open access under the terms of the Creative Commons Attribution License 4.0 International License

\begin{abstract}
The concept of eye tracking is developing rapidly across various disciplines, and this inspired the current study of using this technology for an educational purpose. Eye tracking is a measuring method to either identify the gaze point or relative motion of the eyes to the head. Researchers at NTNU I Ålesund, have used eye-tracking technology in several projects in the field of maritime human factors, but have not explored its potential to improve the process in manufacturing industries and due to lack of knowledge about the potential of eye tracking technology, its prospect in engineering education was also not investigated. This article provides an overview of usability of eye-tracking technology in engineering education (lean manufacturing course) and manufacturing industries. It presents a hybrid of qualitative and quantitative analysis. A case study performed in the industry to investigate the use of eye tracker in the process improvement of the manufacturing field with the help of smart class environment. This article provides a brief description and discussion of lean and eye-tracking technology as well as its possible applications and limitations.
\end{abstract}

Keywords Lean Systems, Ergonomics, Human Factors, Eye Tracking, Engineering Education

\section{Introduction}

In recent years, engineering teaching methods in the universities have changed rapidly, becoming increasingly practical, such that it is common for students to address real-world problems through university - industrial collaboration. Manufacturing industries are eager to cut costs to remain competitive, which creates the appetite for these partnerships. Universities are increasingly develop their curricula to meet such needs through holistic approaches to support local industries to meet the future demand.

From the research work [1] [2], it is evident that learning factory could be an effective solution, a concept of bringing faculty, students, and industries together. Learning factory is a term coined in Penn state university [3], and figure 1 shows the triangular connection between these actors. The benefits of triangular relation are university-industry collaboration, solving a real-world problem, up-to-date on industrial needs and research, bringing theory and practice together, better curriculum and students' engagement, new ideas/ thinking, and finding new talents. The learning factory approach encompasses design, prototype, etc., here we use it more narrowly as a platform to investigate the application of lean principles to improve capacity in the manufacturing environment and to enhance academic curriculum. This allows us to examine the collaboration and group dynamics between these three actors.

Our study takes place in a smart class environment at NTNU I Ålesund. The smart class environment is necessary because it provides students the opportunity to gain familiarity with the process that takes places in the industry. Due to a short course period (5 days), it is difficult to take the students to visit multiple industries. A smart class environment addresses this problem by bringing the industry to the classroom for students to view. This is realized by using technology such as an eye tracker and Go-Pro for video and audio recording, digital learning through YouTube, and web-based course management software Blackboard.

Eye tracking is a measuring method to either identify the gaze point or relative motion of the eyes to the head. An eye tracker is a device that captures real-time video of eye positions and eye movement [4]. The output of the eye tracker is a clear audio and video with a gaze point.

This data has been analyzed both from the point of view of businesses seeking to understand a problem employees face during work and from the point of view of educating students. A problem statement presented to students by industry, to which they respond by coming up with a solution (new design, creative thinking, etc.) that can benefit the industry. The notion of using high-end technology such as eye tracking in the industry for a lean project opens new dimensions for both lean and human-machine interaction research. Both these fields highly stress ergonomics in the workplace as a way to improve a product or productivity.

Lean is a favorite topic in the manufacturing sector. The 
fundamental of lean productivity is striving for perfection through continuous improvement while focusing on eliminating waste and promoting customer value. The lean model aims to create a culture that will create and sustain a long-term commitment to those values in the face of challenges.

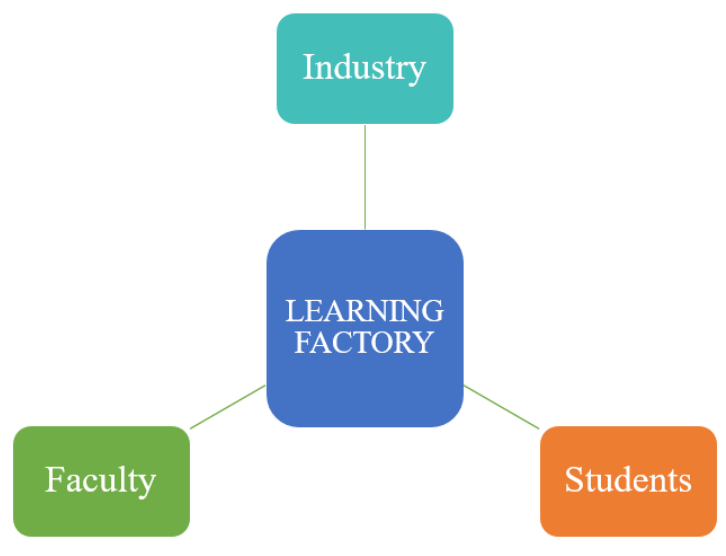

Figure 1. Learning factory

\section{LEAN PRINCIPLES}

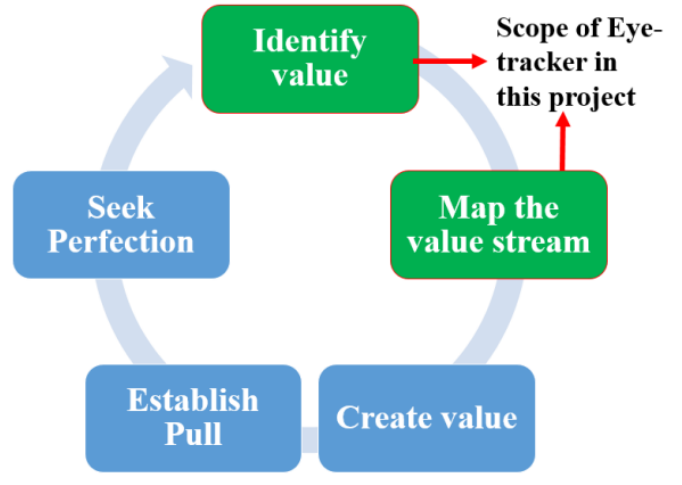

Figure 1. Lean principles and scope of eye tracker

Figure 2 shows lean principles and the scope of the eye tracker in this project. The first principle of lean is to identify value; it is done by Gemba (the Japanese word for, Go and see on the factory floor). An eye tracker can aid in this process through the identifying value (lean waste, ergonomic issues, the efficiency of plant layout, and standard operating procedure (SOP)). The second principle is mapping the value stream, which involves time measurement, process mapping, flowchart, etc. Usually, time measurement requires two persons one to register the sequence of the process and the other to record time. Since the output of an eye tracker provides us with video, this simplifies the process of collecting data. In comparison, less the human resource it is less vulnerable to human error. These videos help with re-testing and as a video tutorial for students to learn.

\section{Objective}

The research objectives are classified into two categories.

The academic objective includes testing the implementation of problem-based learning in academic practice and the applicability of eye tracking as a tool for problem-based learning in the manufacturing sector.

The business objective is to improve production capacity by eliminating waste and integrating lean principles into the partnering firms work culture.

This case study was conducted at XYZ industry (changed on industry request) in the spare part after-sales department. The company manufactures an air compressor, and primary market is marine and power plants. NTNU is a university with an international focus, and campus Ålesund is located at the heart of marine hub; known for its shipbuilding and their related manufacturing industries. NTNU offers lean courses, and the faculty has frequently engaged in industrial collaboration as a part of establishing an eco-system to create learning factory. The first author was a student at NTNU I Ålesund during this project; this article is a part of thesis work written for the fulfillment of master degree and a contribution to improving university's education system.

This article is the revised and extended version of the conference paper [5] on engineering education research, which discussed the new approach of problem-based learning in the lean course through the smart class environment using an eye-tracker as a learning tool. This is a part of our ongoing research towards creating a learning factory at NTNU I Ålesund to upgrade the curriculum and improve the use of technology as a tool in an educational setting.

\subsection{Literature Review}

Eye tracking studies of electronic chip manufacturing show that the scan paths of good inspectors have a systematic pattern of logical skimming with high accuracy and speed. Comparatively, the novice has more extended eye fixation [6]. This consumes time, a waste of resources, which violate lean principles and stresses the operator's eyes, which violate ergonomic principles. [7] Paper suggests an eye tracker can be used during visual inspection for predictive analyses and identifying systematic patterns, train beginner. This systematic pattern helps us to create a standard operating procedure, which is a crucial lean step to reduce variation and defects. These researchers [8] have done more insightful research on visual search strategy in industrial inspection field related to eye movement.

Lean principles identify searching for tools, material or other items as waste. Researchers [9] have explained that the visual search strategy is effective in reducing searching, and that eye movement is used as a feedback mechanism and validation of a new search strategy by the lean practitioner. A survey on eye tracker application in various fields conducted by [10] suggests it can be used in the 
traditional diagnostic application, interactive applications and different approaches such as selective and gaze-contingent.

The article has discussed problem-based learning, and the researcher has dealt with how increasing student activity and reducing lecturing will improve the learning outcomes [11]. In the 1960s, Barrows and Tamblyn developed problem-based learning for McMaster University in Hamilton [12]. Initially designed for the medical school program have found its way into other education programs after its accomplishment. A student-centered educational method framed to address students' lack of ability to apply knowledge in the real world [13].

PBL (Problem based learning) endorses the uses real-world problems to teach the students, in contrast to traditional teaching method of presentation of concepts, principle, facts, followed by assignments and examination [14] [15]. It provides an opportunity for group work, critical thinking, communication, and it paves the path to develop desired skills for life-long learning [16]. The process takes place in small groups of learners, and all learners have a role to play. The tutor's role is to assist, guide, and supervise; these are the ways to facilitate the learning process [17].

The lecture-based traditional method lacks these qualities of problem-based learning [14], which we try to address in our learning factory. Learning factory is a physical facility focuses to solve a real-world problem of manufacturing industry [18]. The industry and tutors prepare the resources and problem statements. To enhance the learning experience, the smart class environment was introduced in engineering education. These recent technologies $(3 \mathrm{G}, 4 \mathrm{G}$, interactive learning, audio, and video, etc.) create a means for knowledge acquisition [19].

The lean system is aimed at providing methods and techniques to create a lean solution from the start, resulting in more value and less wastes across the value stream [20]. Lean design optimizes the development process through rapid learning cycles to build and test multiple concepts early [21]. The process involves managing the knowledge value stream, systematic problem solving with an analysis of the trade-offs between various options [22], and generating solutions generated from ideas filtered by systematic innovation methods [23].

Finding from the literature review suggest that, there are no previous studies similar to the current study. Therefore, there will be no comparison of the results with other studies.

The rest of this paper arranged as follows: section 3 presents materials and methods of the project. Section 4 presents results and discussion. Section 5 presents the conclusion and direction for future research based on this project.

\section{Materials and Methods}

A hybrid of both quantitative (controlled experiment) and qualitative methods was followed in that the method includes interviews, observation, pep talks, workshop, lean games, kaizen activity, and brainstorming. The qualitative case study method used here follows this article [24].

A case study performed in the industry to investigate the use of an eye tracker in the process improvement of the manufacturing field. In contrast to the traditional approach, a new approach is proposed this includes the physical facility like learning factory (Lab) and industry for research work of wearable eye tracker. Lectures and theories of lean principles are presented in smart class environement, with theaching method of problem based learning.

\subsection{Participant and Equipment}

The participants are six students from the university and six employees from the company participated in the study. Students have completed fundamentals of lean principles in the previous semester. While the manager who is also a participant is, a lean practitioner but for the rest of the employees, a workshop has been conducted to educate about lean principle and eye-tracking technology.

Students and employees are at different experiential and educational level, but this helps both employee (managers, operator, etc.) and students interact with each other and learn through group dynamics.

Figure 3 shows the use of eye tracker by a participant during the study.

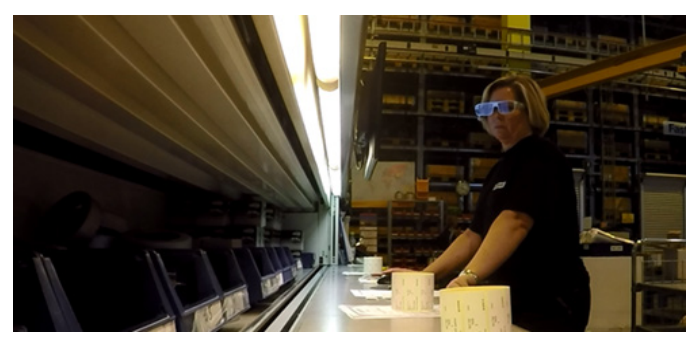

Figure 3. Employee with eye tracker

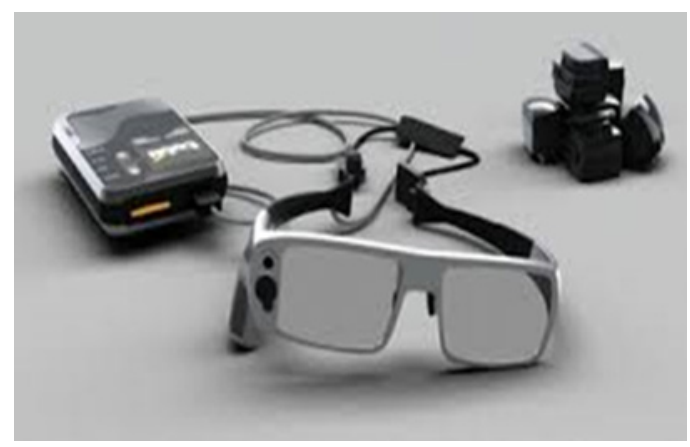

Figure 4. Wearable eye tracker

Figure 4 presents wearable eye tracker intended to capture viewing pattern of humans in the real-world 
environment. The equipment required calibration before using it. The wearable eye tracker is a mobile system used to collect data (i.e., to record video and audio, with gaze point) from a user's perspective. The data is stored in a recoding unit and retrieved for further processing using Tobii pro glass controller software. The picture below shows the use of an eye tracker by the participant (employee) during the study.

In this project, eye tracker was used as a tool to determine the point of gaze and to understand the usability of eye tracker in a manufacturing environment. The experiment will provide us with workers view patterns, their difficulties, an understanding of the manufacturing process and time each step in it takes.

Some of the limitations of this case study are that eye trackers full potential is not utilized, such as snapshot and real-world mapping tools. Mapping provides important analysis and exhibit results with insights. The experiments are performed under a controlled environment to record. Since the circumstances in the real world are not controllable. The scenario has been established during the study in before, and after case, data has been recorded.

\subsection{Procedure}

According to the article [24], the procedure for getting insight into a project, the following steps are modified and adopted to the needs of the experiment.

1. Students on-site visit to the factory and record the present state of the workplace (current state mapping),

2. Students, workers, and managers gather for workshop and brainstorming, with the professor, and research assistant being facilitators.

3. Planning the potential improvements systematically (future state mapping),

4. Interview and discussion (with students, workers, and managers),

5. Investigation of outcomes after implementation.

The preparation and procedure was a simple task as a team of students from university worked with the company employees whose work activity was the focus of data collection. The employees were educated about lean principles and implementation of lean tools. The students, who had already completed lean courses, received a detailed introduction to the department, the industry, process, activity, work culture and the problem their project would seek to solve. The author acted as a moderator in these exchanges of information collected eye tracker data during the on-site visit.

During the workshop session, the collected data were analyzed systematically with employees, with the students acting as customers to evaluate the value and non-value added activity. This helps the employees to see from customer's point of view. The consensus was reached on what constituted non-value added activity, as that is our primary concern.

A brainstorming session followed in which both students and employees considered how to eliminate or minimize non-value added operations. This provided participants with problem-based learning and acted as a platform for group dynamics.

At the end of this session, a possible future state was mapped for further improvement in the participating department. This part of the course help's students to improve their problem-solving skills, teamwork, and project management.

The final step in that day's activities was an interview, group discussion and a 10-question survey of the students to evaluate their learning outcomes. The findings appear in the next section and the paper's conclusion.

\section{Results and Discussion}

This section explains examples of how eye trackers work and how the participants used to solve problems. It then discusses the results of the experiment. These findings are in keeping with the understanding that an eye tracker can function in a lean process by identifying value and mapping the value stream.

\subsection{Identifying Value}

Figure 5 presents the moments of recording eye tracking data during workers operation. The red dot represents the pupil movement from one place to another; this assists us to see how the employee does certain activities. The employees are questioned systematically about these activities, that respective employee prepares a table of value added, and non-value added activities. During this categorizing, students act as customers by describing what they see as value adding activities.

For every single order, the employee was trying to find a reference number on a label and crosscheck the same in the order paper multiple times. This is a time-consuming process as the number of order increases the time wasted identifying reference numbers also increased.

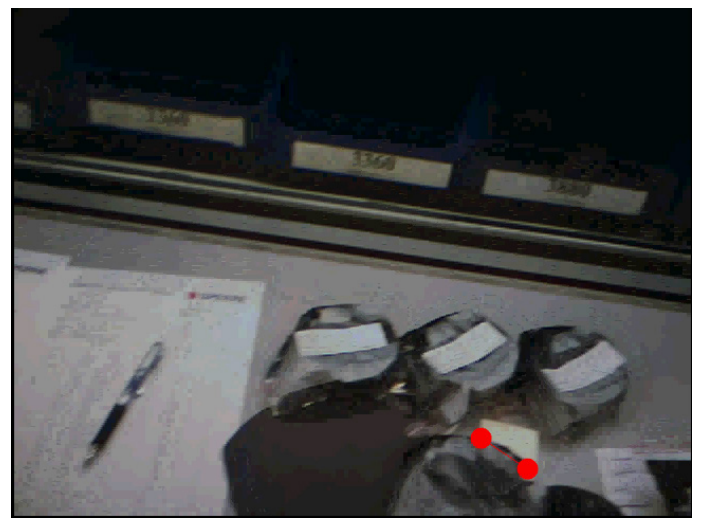

Figure 5. Eye tracking during time study 


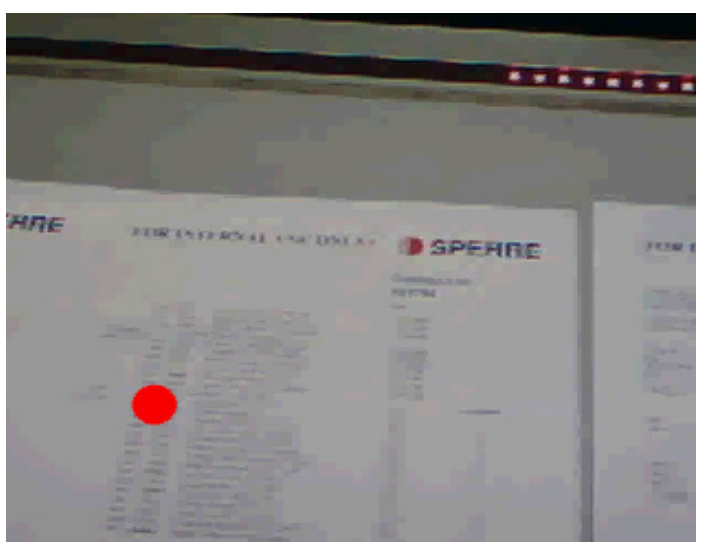

Figure 6. Gaze point during reference number

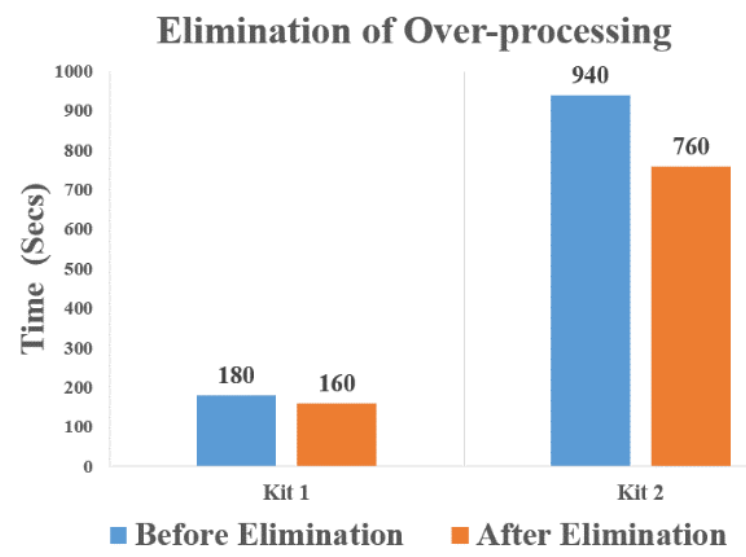

Figure 7. Result of elimination of over processing

Figure 6 shows the employees struggle to find those reference numbers every time due to small text. The student's point of view revealed that the reference number identification could be avoided and an alternative solution could be used to save time. In the end, the employee understood it is a non-value added activity and thus eliminated it. This is one example of how eye tracker revealed even small details could be noted using this technology and elimination of that step saved a lot of production time.

Figure 7 shows the amount of time saved after eliminating the time seeking the reference number as well as the fact that time wasted or saved was directly proportional to the number of items in the order. In this case, we can see for the particular kit it saved 20 seconds, and for another, it saved 180 seconds during production for kit 2 . In both cases, this was a significant effect, given the overall production time.

\subsection{Mapping Value Stream}

\section{Workflow Mapping:}

The eye tracker was used to visually map the production flow and shows the current state of processes. Since the eye tracker has the ability to record both the audio and video. It can be used to demonstrate the process to the participant by walking through the process. The following cross-functional flowchart (figure 8) is prepared as the employee explains the process flow in the video.

This cross-functional flowchart helps in classifying and differentiating various activities, which in terms helps us to concentrate more on the problematic area. Initially, it starts with an order from the customer followed by the rest of the actions, with respect to the product family. The only difference between two-product families is packing method either by lamination or with boxes. This flowchart helped us to realize that it is required to standardize product thus lamination has been reduced drastically. The industry has mostly adopted kitting in the box because of this.

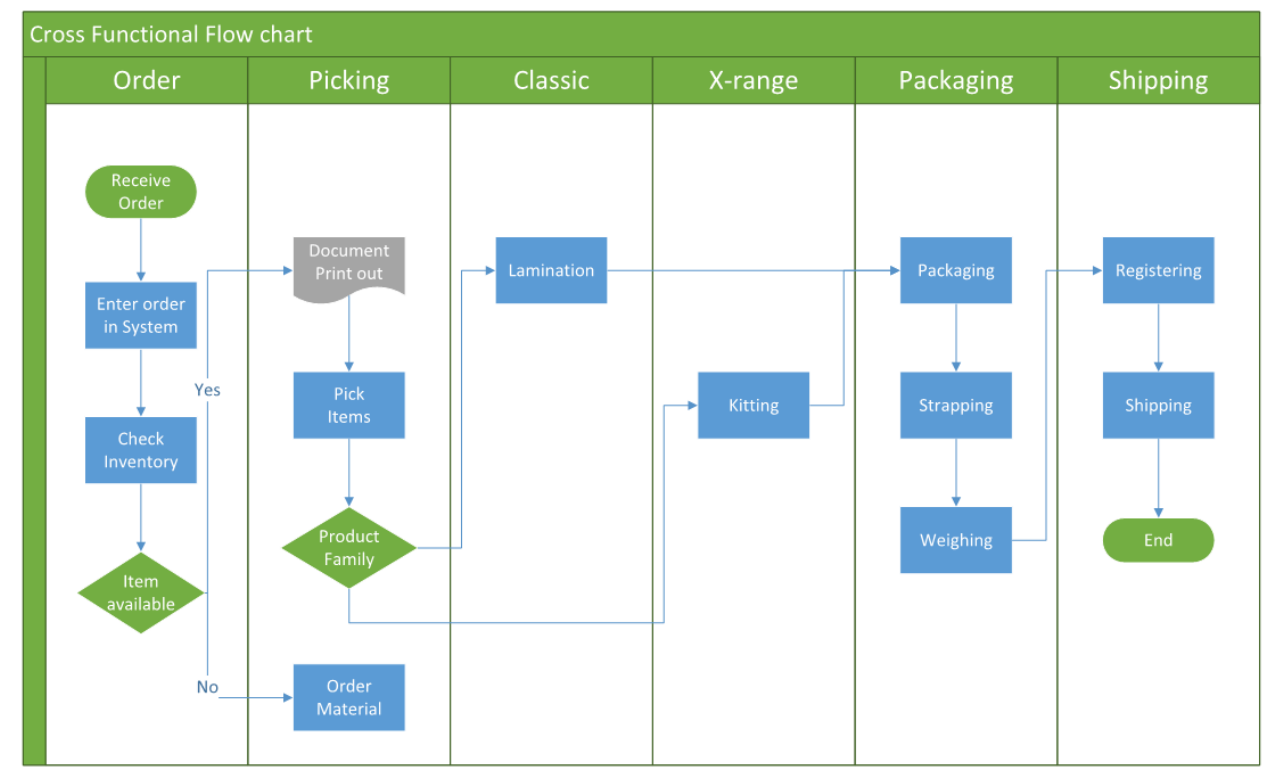

Figure 8. Process flow chart 


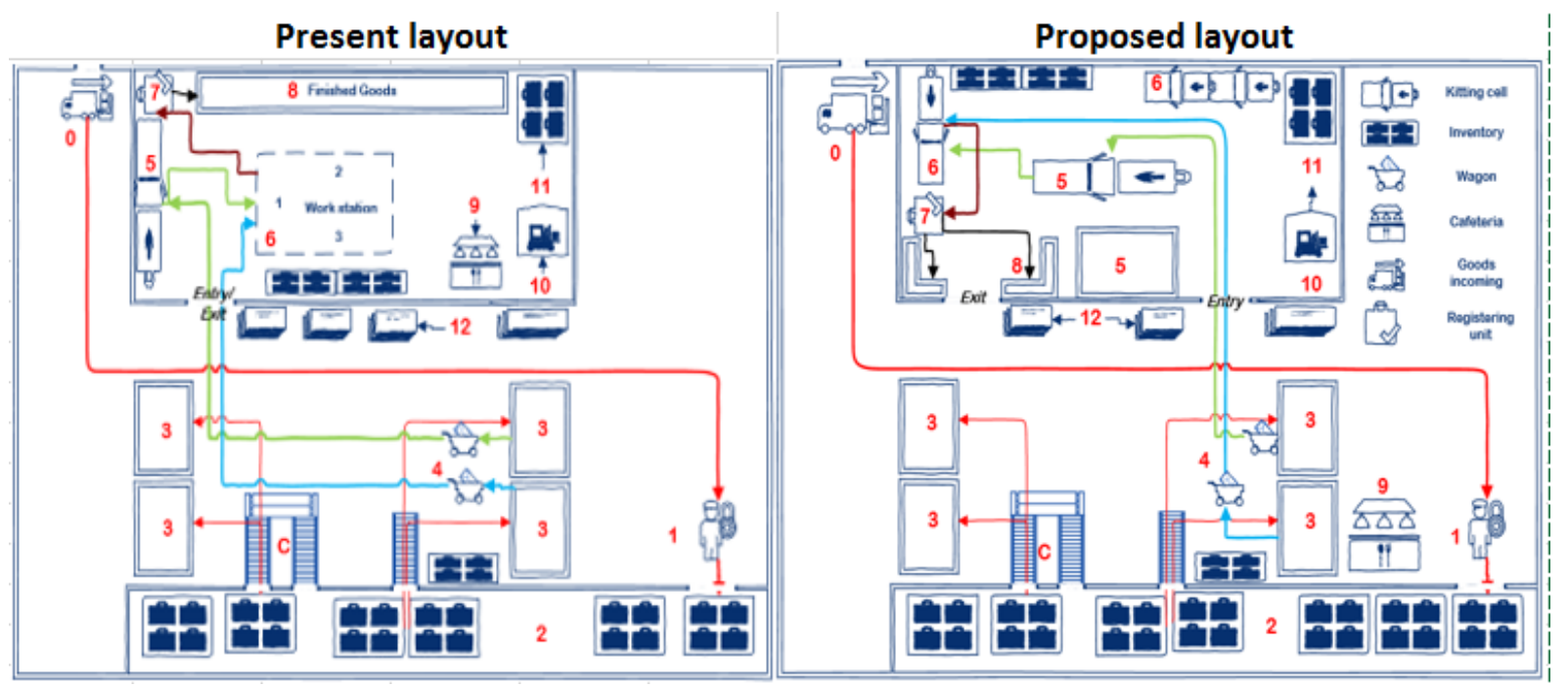

Figure 9. layout modification
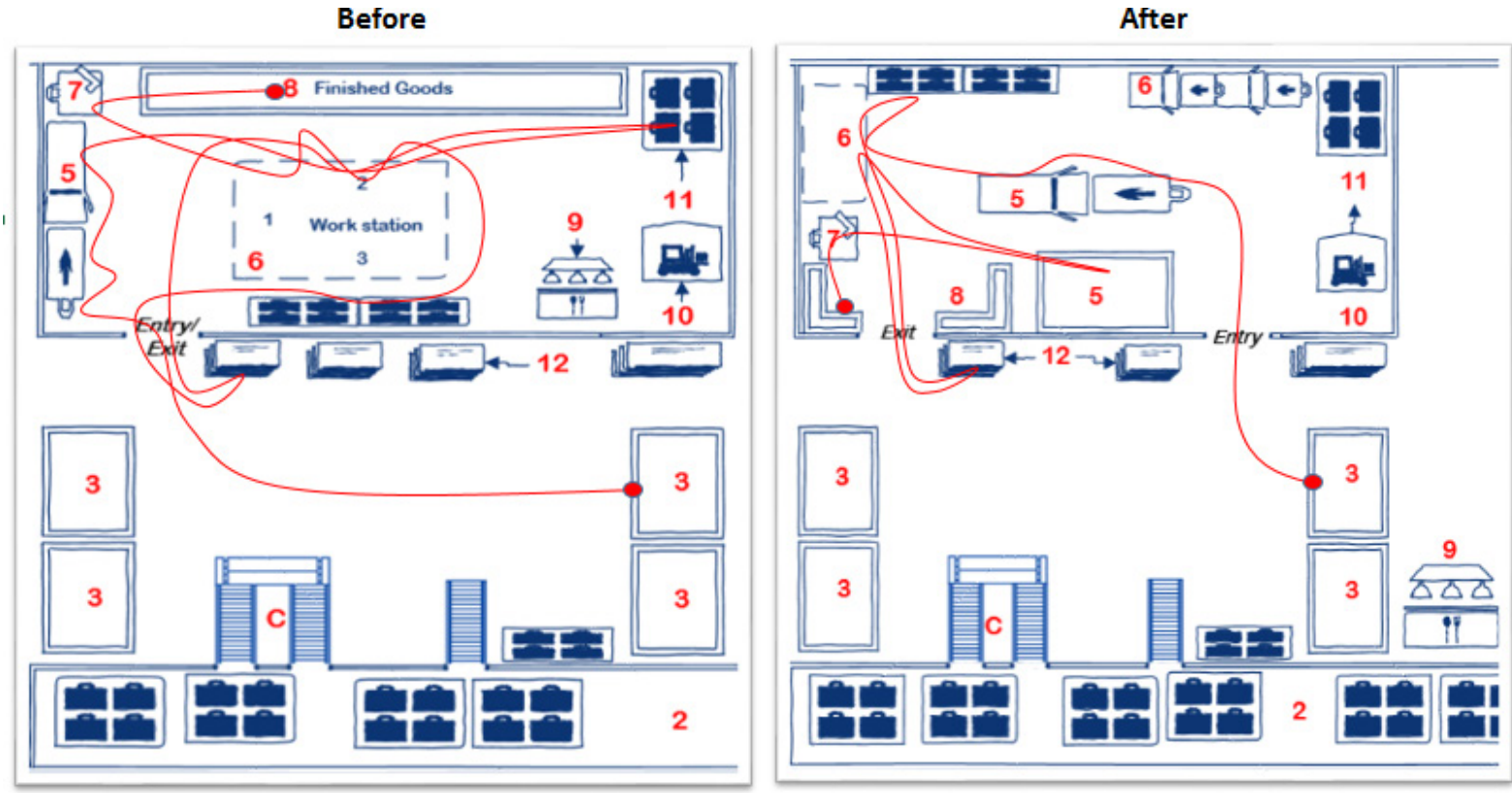

Figure 10. Spaghetti diagram

Plant Layout Design and Spaghetti Diagram

The eye tracker was also used for designing and reorganizing the plant layout, a critical lean tool in process improvement. This provides both the students and employees of the organization the in-depth knowledge about the lean principles. It is important because layout modification involves a lot of lean tools, such as Kanban (scheduling system), inventory, and flexibility. The complete outline of the layout of the department, figure 10 was drawn using the data from the video of the eye tracker. The discussion helped us to eliminate waste and visualize new layout according to operational flow. The present layout and proposed layout are shown in figure 10.

After implementation, for verification, the time taken in the new layout is compared to the time taken in the original.
The new layout is validated using a Spaghetti diagram, as shown in figure 11. The walking distance of the old layout and new layout were compared, showing new layout reduced operators' motion considerably. The new layout also provides the flexibility that improves efficiency. The eye-tracking tool was the only one required to implement these changes.

The activities that are represented in figure 9 of layout modification are represented from zero to 11 . The colored lines signal the following: Red lines: receiving and loading activities. Green lines: workflow of classic kits. Blue lines: $\mathrm{x}$-range kits, brown represents strapping, weighing and registering activities. Black lines: storing finished goods for departure.

1. Receiving goods from suppliers 
2. Load to warehouse

3. Load to Paternoster (Supermarket)

4. Picking

5. Load for lamination/ cutting

6. Workstation

7. Strapping, Weighing and Registering

8. Finished goods

9. Cafeteria

10. Large kit packaging

11. Store for packing items

12. Carton tray store

A time study on the entire process including all the steps was done on both layouts. The same production order was used to create a comparison to determine the time taken for a complete order with each layout in real time.

Table 1. Layout comparison

\begin{tabular}{|c|c|c|}
\hline Layout & Time $(\mathrm{Sec})$ & $\begin{array}{c}\text { Walking distance } \\
\text { (meter) }\end{array}$ \\
\hline Previous layout & 720 & 45 \\
\hline Present Layout & 570 & 32 \\
\hline Difference & 150 & 13 \\
\hline
\end{tabular}

Table 1 shows the result of the layout comparison and figure 10 shows spaghetti diagram, it is clear that the present layout saves $150 \operatorname{secs}(21 \%)$ because of the flow of the plant, machine arrangement, and reduced traffic in the entry and exit. The walking distance has also reduced from 45 to 32 meter (i.e., $28 \%$ ) for that specific production order.

\section{Ergonomic}

During routine task it has been identified from the eye-tracking study one of the operator was not comfortable with the workstation due to operator's height. In addition, the operators took a long time to finish packing due to picking of raw material from different places. This promoted the company to change all the workstation optimized for individual needs. Therefore, a multi-functional workstation was provided with the required material at the right quantity (material pre sentation).

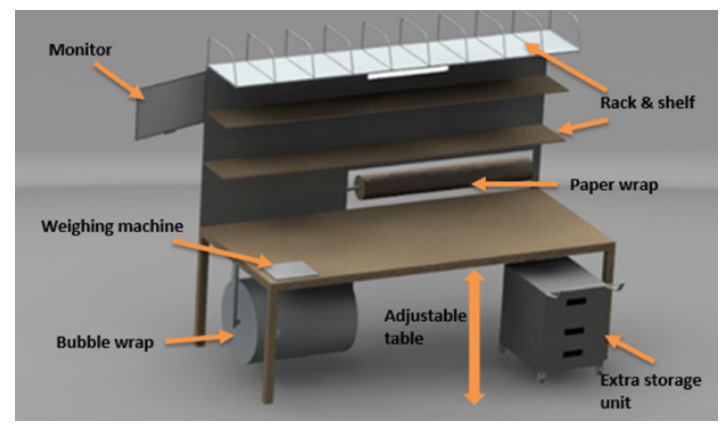

Figure 11. Ergonomic workstation

Figure 11 presents the ergonomic workstation, while figure 12 presents the comparison of before and after case scenario of the old and new workstation. It is evident that new workstation saves 48 seconds for the same kit after implementation.

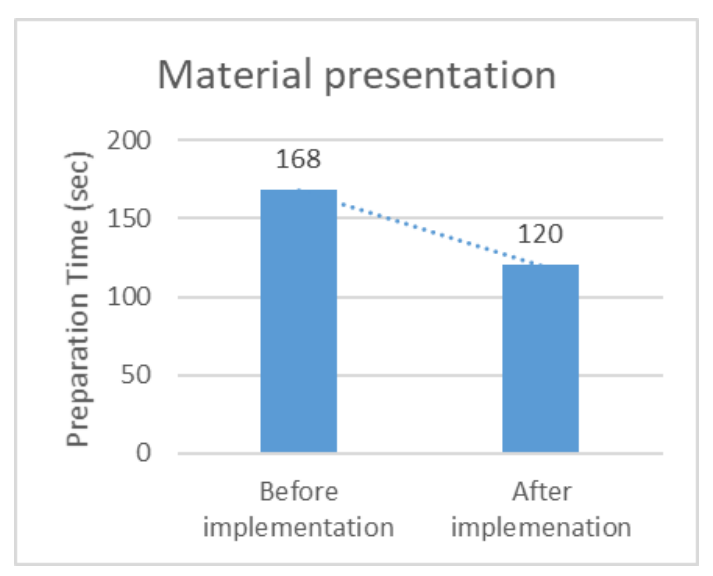

Figure 12. Time study of workstation

\subsection{Overall Result}

The overall results are described from both a business and an education perspective.

From the business perspective, the overall performance has improved just by eliminating waste. This was achieved without any capital investment by the company and thus is an unmitigated positive. So the outcome has compared to the time taken for producing a particular product before and after case scenario.

As figure 13 and 14 reflect, it took 9:36 mins to produce a kit in the before case scenario and 8:24 mins in after case scenario. Thus production time was reduced by $13 \%$.

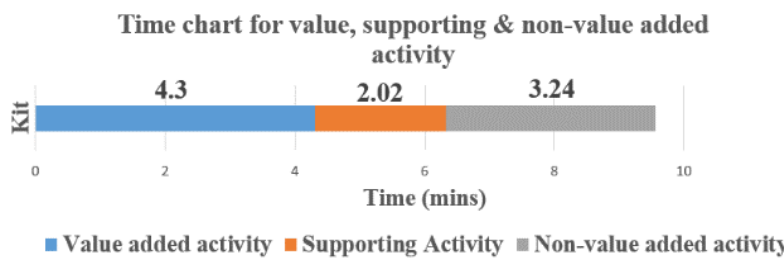

Figure 13. Before case scenario (business outcome)

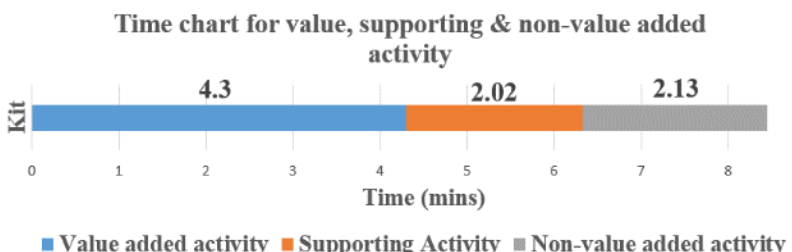

Figure 14. After case scenario (business outcomes)

Implementing lean principle in the work culture is also a part of the project. Table 2 shows the eye tracker technology applicable for lean tools in the Manufacturing industry. 
Table 2. Eye tracking tool in lean tools

\begin{tabular}{|c|c|}
\hline Lean Tools & Applicability \\
\hline Value Stream Mapping & $\mathrm{x}$ \\
\hline Process Mapping & $\mathrm{x}$ \\
\hline Group Technology & $\mathrm{x}$ \\
\hline Cellular Manufacturing & $\mathrm{x}$ \\
\hline Point of use Storage & $\mathrm{x}$ \\
\hline Material Presentation & $\mathrm{x}$ \\
\hline Ergonomic & $\mathrm{x}$ \\
\hline Standardized Work & $\mathrm{x}$ \\
\hline Layout Design & $\mathrm{x}$ \\
\hline Line Balancing & $\mathrm{x}$ \\
\hline Spaghetti Diagram & $\mathrm{x}$ \\
\hline Bottleneck Analysis & $\mathrm{x}$ \\
\hline Kanban & $\mathrm{x}$ \\
\hline Kaizen & $\mathrm{x}$ \\
\hline $6 \mathrm{~S}$ & $\mathrm{x}$ \\
\hline Visual control & $\mathrm{x}$ \\
\hline
\end{tabular}

The author has performed several workshops for the students after the implementation of these lean tools so that they can understand the connection between theory and practice and the difficulties involved in solving the real world problem.

From an education perspective, this test does not provide any empirical data, for assessment of students' performance. On another hand, the qualitative result, i.e., feedback from the students shows the approach had a positive effect on them. Most students said that the project helped them develop teamwork and real-life example makes it easier to learn the lean principles. Student engagement is more in the learning factory platform and with the smart class environment when compared to theory-based static course content.

On the other hand, students were less positive about the use of the eye-tracker. They pointed out that it is expensive, and difficult to use. They pointed out that they had to be educated on this particular hardware and software, which is a time-consuming process.

\section{Conclusion and Future Work}

This paper presents, a case study to problem-based learning through the smart class environment using an eye tracker as a tool. The eye tracker was used to collect data and assist the participant in realizing lean principles such as identifying the value and mapping the current state. Workshop and brainstorming sessions were performed with students who have already completed the lean course. Students were used to evaluate and compare the traditional and new approach to improve the course curriculum at the university.
The method used here creates more interaction and synergy between employees and students, where both can learn at the same time. It is expected that this approach provides an opportunity for students, employees, and others to access these videos, through a virtual smart class environment from anywhere, at any time to educate themselves at their own pace, and offer a solution to the problem. It also increases the students' understanding of theory as a whole and provides them real-world experience that prepares them for more industrial projects.

Compare to theory and lectures alone; real-world application engenders more enthusiasm and involvement among students. Also, the feedback from the students shows that they were motivated by the real-world project. The industrial partner benefited by their business results through the implementation of lean principles in their work culture and educating their employees. The business results have shown the teamwork between students and employees facilitated problem-solving to increase productivity without any significant investment on the part of the company.

In summary, the approach of problem-based learning with the help of an eye tracker is useful and has great potential in engineering education settings. However, there are several shortcomings of eye tracker technology such as cost, the difficulty of use, company location and mobility of students and employees. In the future, we aim to identify improvements in the smart classroom environment and further research is required to have a better understanding of the potential or possible challenges in academic. Similarly, more research is required to observe the potential application of eye-tracking technology in the manufacturing sector. This technology unlocks new research area for industrial training, skill and knowledge transfer. During the training period, best practice can be established using these videos and this also brings out implicit knowledge of skilled employees. For example, eye-tracking technology was used in packing of kits to identify best practice, understand the subsequent process, visual and work skills of the experienced employee. These systematic processes are documented after scrutiny by the students for creating a standard operating procedure (best practice). This also reduces training time of the new trainees in production, assembly and quality lines. Further, safety and ergonomic at manufacturing site are also potential field for future study.

\section{REFERENCES}

[1] E. Abele, G. Chryssolouris, W. Sihn, J. Metternich, H. ElMaraghy, G. Seliger, G. Sivard, W. ElMaraghy, V. Hummel, M. Tisch og S. Seifermann, «Learning factories for future oriented research and education in manufacturing,» CIRP Annals - Manufacturing Technology, 2017. 
[2] O. J. Mork, I. E. Hansen, K. Strand, L. A. Giske og P. S. Kleppe, «Manufacturing Education- Facilitating the Collaborative Learning Environment for Industry and University,» $i$ Procedia CIRP, 2016.

[3] T. P. S. University, «Bernard M. Gordon learning factory,» 2015. [Internett]. Available: http://www.lf.psu.edu/.

[4] «Wikipedia: Eye tracking,» 2017 November 15. [Internett]. Available: https://en.wikipedia.org/wiki/Eye_tracking.

[5] K. K. Vijayan, I. e. Hansen, S. Komandur og O. j. Mork, «Lean design of spare part kit production cell: A case study from Sperre industry AS,» i SEFI, Tampere, 2016.

[6] J. W. Schoonard, J. D. Gould og L. A. Miller, «Studies of Visual Inspection,» Ergonomics, pp. 365-379, 1973.

[7] M. V. Yousefi, E. Karan, A. Mohammadpour og S. Asadi, «Implementing Eye Tracking Technology in the Construction Process,» i 51st ASC Annual International Conference Proceedings, Texas, 2015.

[8] E. D. Megaw og J. Richardson, «Eye Movements and Industrial inspection,» Applied Ergonomics, pp. 145 -154, 1979.

[9] M.-J. J. Wang, S.-C. Lin og C. G. Drury, «Training for strategy in visual search,» Industrial Ergonomics, pp. 101-108, 1997.

[10] A. T. Duchowski, «A Breadth-First Survey of Eye Tracking Applications,» Behavior Research Methods, Instruments, \& Computers, pp. 455-470, November 2002.

[11] L. Jensen, «Does increasing student activity and reducing lecturing improve learning,» i SEFI, Birmingham, 2014.

[12] H. S. Barrows og R. M. Tamblyn, Problem-based learning: ing: An approach to medical education., New York: Springer, 1980

[13] W. Hung, "All PBL Starts Here: The Problem,» The Interdisciplinary Journal of problem based learning, 1 January 2016.

[14] W. Hung, «Theory to reality: A few issues in implementing problem-based learning,» Educational Technology Research and Development, p. 529-552, 17 March 2011.

[15] Illionis CITL, «Teaching and Learning (Problem based learning),» [Internett]. Available:

http://citl.illinois.edu/citl-101/teaching-learning/resources/t eaching-strategies/problem-based-learning-(pbl). [Funnet 23 September 2018].

[16] B. J. Duch, S. E. Groh og D. E. Allen, The power of problem-based learning, Virginia: Stylus, 2001.

[17] H. G. Schmidt, J. I. Rotgans og E. H. Yew, «The process of problem-based learning: What works and why,» Medical Education , p. 792-806, 14 July 2011.

[18] J. S. Lamancusa, J. L. Zayas, A. L. Soyster, L. Morell, Jorgensen og Jens, «2006 Bernard M. Gordon Prize Lecture*: The Learning Factory: Industry - Partnered Active Learning,» Journal for engineering education, 2 January 2013.

[19] A. Alelaiwi, A. Alghamdi, M. Shorfuzzaman, M. Rawashdeh, M. S. Hossain og G. Muhammad, «Enhanced engineering education using smart class environment,» Computers in Human Behavior, pp. 852-856, October 2015.

[20] J. Womack, D. T. Jones og D. Roos, The Machine That Changed the World: The Story of Lean Production, Toyota's Secret Weapon in the Global Car Wars That Is Now Revolutionizing World Industry., New York, 1990.

[21] T. Schipper og M. Swets, Innovative Lean Development: How to Create, Implement, and Maintain a Learning Culture Using Fast Learning Cycles., New York, (2010).

[22] A. P. Ward, Lean Product and Process Development. (2007).

[23] B. S. Huthwaite, the Lean Design Solution: A Practical Guide to Streamlining Design and Development, 2004.

[24] I. E. Hansen, A. Karlsen, O. Mork, M. Mørk og A. Persson, «Planning for waste minimization at a factory floor: A student project to support smarter home production practices,» i SEFI, Tampere, 2016. 